\title{
CLOSURE OF INVERTIBLE OPERATORS ON A HILBERT SPACE
}

\author{
RICHARD BOULDIN
}

(Communicated by Palle E. T. Jorgensen)

\begin{abstract}
Although most of the characterizations of the closure of the invertible operators on a separable Hilbert space do not extend to a nonseparable Hilbert space, this note gives a characterization for an arbitrary Hilbert space that generalizes the separable case in a natural way. The new concept of essential nullity, which facilitates this characterization, should find other applications.
\end{abstract}

\section{INTRODUCTION}

This research is motivated by a theorem proved by Robin Harte in [6]. In order to state that result we recall the following definitions. An element $a$ of the Banach algebra $\mathscr{A}$ is said to be regular provided there is an element $b \in \mathscr{A}$ such that $a=a b a$. We say that $a$ is decomposably regular provided the $b$ in the preceding equation can be chosen to be an invertible element of $\mathscr{A}$. Harte's theorem asserts that a regular element $a$ belongs to the closure of the invertible elements if and only if it is decomposably regular.

Much of the interest in Harte's theorem arises from its application to the Banach algebra $\mathscr{B}(H)$ of (bounded linear) operators on the Hilbert space $H$. For $T \in \mathscr{B}(H)$ we define nul $T$ and $\operatorname{def} T$ to be the cardinal numbers $\operatorname{dim} \operatorname{ker} T$ and $\operatorname{dim} \operatorname{ker} T^{*}$, respectively. It follows from the work of Atkinson [1] that $T$ is regular if and only if $T$ has closed range. We shall prove in the next section that a regular operator $T$ is decomposably regular if and only if nul $T=\operatorname{def} T$. Thus, it follows from Harte's theorem that an operator $T$ with closed range belongs to the closure of the invertible operators $\mathscr{G}$ if and only if nul $T=\operatorname{def} T$. This characterization does not require the underlying Hilbert space to be separable.

When $H$ is separable there is a nice characterization of the closure of $\mathscr{G}$ that is immediate from the main theorem of [3]. The operator $T$ belongs to the closure of $\mathscr{G}$ if and only if nul $T=\operatorname{def} T$ or the range of $T$ is not closed. Regardless of whether $H$ is separable or not, the closure of $\mathscr{G}$ is characterized in [4] but the characterization uses $W^{*}$ algebra existence theorems and

Received by the editors March 3, 1989.

1980 Mathematics Subject Classification (1985 Revision). Primary 47A05; Secondary 47A55.

Key words and phrases. Invertible operator, closure, ring of operators, nonseparable Hilbert space, essential nullity. 
concepts that are difkcult to inferpret conctetely. This note will establish a chareterization that is aqalogous to Harte's theorem and is easy to understand conctetely.

1e $U|T|$ be the usual potar factorization of $T$ and let $E(\cdot)$ be the spectral measure for the nonnegative operator $|T|$. We define ess nul $T$ by the equation

$$
\text { ess nul } T=\inf \{\operatorname{dim} E([0, \varepsilon)) H: \varepsilon>0\}
$$

and we define ess $\operatorname{def} T$ by

$$
\text { ess def } T=\operatorname{ess} \operatorname{nul} T^{*} \text {. }
$$

\section{Main Results}

First we prove the previously mentioned proposition that facilitates the application of Harte's theorem to the Hilbert space case.

1. Proposition. Suppose $T \in \mathscr{B}(H)$ has closed range. The operator $T$ is decomposably regular if and only if $\operatorname{nul} T=\operatorname{def} T$.

Proof. If $\operatorname{nul} T=\operatorname{def} T$ then there is a linear isometry $V$ of $(T H)^{\perp}$ onto $\operatorname{ker} T$. Let $B$ coincide with $V$ on $(T H)^{\perp}$ and on $T H$ let it be the inverse of $T$ restricted to $(\operatorname{ker} T)^{\perp}$. Then $T B T=T$ holds.

Assume that $T B T=T$ holds for an invertible operator $B$. Since $T B$ is idempotent and $T H=T B H$, we see that $H=T H \oplus \operatorname{ker} T B$. It follows from this equation that

$$
\operatorname{dim} \operatorname{ker} T^{*}=\operatorname{dim}(T H)^{\perp}=\operatorname{dim} \operatorname{ker} T B=\operatorname{dim} \operatorname{ker} T .
$$

We shall need the next lemma in the proof of the main theorem.

2. Lemma. Let $T=U|T|$ be the usual polar factorization and let $V$ be the isometry obtained by restricting $U$ to the closure of the range of $T^{*}$, denoted $\left(T^{*} \tilde{H}\right)^{*}$, and considering $(T H)^{-}$to be the range of $V$. Let $E(\cdot)$ and $F(\cdot)$ denote the spectral measures of $|T|$ and $\left|T^{*}\right|$, respectively. Then on the obvious subspages we have $\left|T^{*}\right|=V|T| V^{*}$ and $F(\mathscr{I})=V E(\mathscr{I}) V^{*}$ for any interval $\mathscr{I}$ contained in $(0, \infty)$.

Proof. Note that $T T^{*}=U|T|^{2} U^{*}=U\left(T^{*} T\right) U^{*}$. Since the square root of an operator is the limit of polynomials in the operator (see [5, Problem 95]), we at $n T V^{*}=\left|T^{*}\right|$. The final assertion follows from the equations

$$
\begin{aligned}
& \left|T^{*}\right|=V|T| V^{*}=V\left(\int_{\mathscr{I}} t d E(t)\right) V^{*}, \\
& \left|T^{*}\right|=\int_{\mathscr{g}} t d V E(t) V^{*}
\end{aligned}
$$

and the for the last equation characterizes $F(\cdot)$.

Nom werm ste and prove our main result. 
3. Theorem. The operator $T$ belongs to the closure of the invertible operators $\mathscr{G}$ if and only if ess nul $T=\operatorname{ess} \operatorname{def} T$.

Proof. Because $\operatorname{dim} E([0, \varepsilon)) H$ is a discrete valued nondecreasing function of $\varepsilon$, we conclude that there is a positive $\gamma$ such that $\operatorname{dim} E([0, \varepsilon)) H=\operatorname{ess} \operatorname{nul} T$ for $0<\varepsilon \leq \gamma$. Similarly we get $\operatorname{dim} F([0, \varepsilon)) H=\operatorname{ess} \operatorname{def} T$ for $0<\varepsilon \leq \gamma$ and so we assume that $\operatorname{dim} E([0, \varepsilon)) H=\operatorname{dim} F([0, \varepsilon)) H$ for $0<\varepsilon \leq \gamma$. Define $R(\varepsilon)$ to agree with $|T|$ on $E([\varepsilon, \infty)) H$ and to agree with $\varepsilon I$ on $E([0, \varepsilon)) H$; define $U(\varepsilon)$ to agree with $U$ on $E([\varepsilon, \infty)) H$ and on $E([0, \varepsilon)) H$ let it agree with $V$, where $V$ is an isometry of $E([0, \varepsilon)) H$ onto $F([0, \varepsilon)) H$. Using the final conclusion of the lemma and the fact that

$$
U E([0, \infty)) H=U(|T| H)^{-}=(T H)^{-}=\left(\left|T^{*}\right| H\right)^{-},
$$

we see that $U(\varepsilon)$ is one-to-one and onto; thus, $U(\varepsilon)$ is invertible. Clearly $R(\varepsilon)$ is invertible and it is straightforward to see that

$$
\|U R-U(\varepsilon) R(\varepsilon)\| \leq 2 \varepsilon .
$$

This proves that $T$ belongs to $\mathscr{G}^{-}$.

Assume that $A_{j}$ is a sequence of invertible operators such that $\left\|T-A_{j}\right\| \rightarrow 0$ and, for the sake of a contradiction assume that ess nul $T \neq \operatorname{ess} \operatorname{def} T$. Replace $T$ with $T^{*}$, if necessary, so that ess nul $T>\operatorname{ess} \operatorname{def} T$. Note that $\left\|T^{*} T-A_{j}^{*} A_{j}\right\| \rightarrow 0$. By the continuity of square roots (see [2, Theorem 2]), we conclude that $\left\||T|-\left|A_{j}\right|\right\| \rightarrow 0$.

Choose $\gamma>0$ such that

$$
\operatorname{dim} E([0, \varepsilon)) H=\operatorname{ess} \operatorname{nul} T, \quad \operatorname{dim} F([0, \varepsilon)) H=\operatorname{ess} \operatorname{def} T
$$

for $0<\varepsilon \leq \gamma$. Let $H_{\gamma}=E([\gamma, \infty)) H$ and define $B$ on $H_{\gamma}$ to agree with the inverse of $|T|$ restricted to $H_{\gamma}$, denoted $\left(|T| \mid H_{\gamma}\right)^{-1}$; let $B$ be zero on $\left(H_{\gamma}\right)^{\perp}$. Note that $T B=U E([\gamma, \infty))$ and that $U\left|A_{j}\right|(B+1 / j)$ converges to $T B$. Let the polar factorization of $U\left|A_{j}\right|(B+1 / j)$ be denoted $W_{j} R_{j}$ where $R_{j} \geq 0$; note that $W_{j}$ is unitary and $\left\|R_{j}-W_{j}^{*} U E([\gamma, \infty))\right\| \rightarrow 0$. Choose $j$ sufficiently large that

$$
\left\|R_{j}-W_{j}^{*} U E([\gamma, \infty))\right\|<\frac{1}{3}
$$

Because the dimension of $\operatorname{ker} W_{j}^{*} U E([\gamma, \infty))=E([0, \gamma)) H$ exceeds $\operatorname{dim} W_{j}^{*} F([0, \gamma)) H$ there exists a unit vector $f$ in $E([0, \gamma)) H$ that is orthogonal to $W_{j}^{*} F([0, \gamma)) H$. This follows from the observation that $F([0, \gamma)) W_{j}$ restricted to $E([0, \gamma)) H$ must have nontrivial kernel. The next equations follow primarily from the lemma:

$$
\begin{aligned}
\left(W_{j}^{*} U E([\gamma, \infty))\right)^{*} & =\left(W_{j}^{*} U E([\gamma, \infty)) U^{*} U\right)^{*}=\left(W_{j}^{*} F([\gamma, \infty)) U\right)^{*} \\
& =U^{*} F([\gamma, \infty)) W_{j} .
\end{aligned}
$$


Thus, the kernel of $\left(W_{j}^{*} U E([\gamma, \infty))\right)^{*}$ is $W_{j}^{*} F([0, \gamma)) H$. From the inequality (*) and the fact that $U^{*} F([\gamma, \infty)) W_{j}$ is a partial isometry we get

$$
\frac{1}{3}>\left\|R_{j} f-W_{j}^{*} U E([\gamma, \infty)) f\right\|=\left\|R_{j} f\right\|
$$

and

$$
\frac{1}{3}>\left\|R_{j} f-U^{*} F([\gamma, \infty)) W_{j} f\right\| \geq\left\|U^{*} F([\gamma, \infty)) W_{j} f\right\|-\left\|R_{j} f\right\| \geq \frac{2}{3} .
$$

This contradiction proves that ess nul $T=\operatorname{ess} \operatorname{def} T$.

Note that Theorem 3 extends Harte's theorem in the Hilbert space case since ess nul $T=\operatorname{nul} T$ and ess $\operatorname{def} T=\operatorname{def} T$ when $T$ has closed range.

The next lemma provides an alternative characterization of ess nul $T$. We shall use the result in the proof of Theorem 5 .

4. Lemma. Let $\varepsilon>0$ and cardinal number $\beta$ be given. We have $\operatorname{dim} E([0, \varepsilon)) H=\beta$ if and only if the inequality $\left\|T \mid H_{\varepsilon}\right\|<\varepsilon$ implies that $\operatorname{dim} H_{\varepsilon} \leq \beta$ and equality is achieved in the last inequality for some choice of $H_{\varepsilon}$.

Proof. If $\operatorname{dim} E([0, \varepsilon)) H=\beta$ then we choose $H_{\varepsilon}$ to be $E([0, \varepsilon)) H$ and we need only prove that there is no subspace $H_{0}$ with dimension higher than $\beta$ and $\left\|T \mid H_{0}\right\|<\varepsilon$. For the sake of a contradiction assume that $\operatorname{dim} H_{0}=\alpha>\beta$. Since

$$
\operatorname{dim} E([0, \varepsilon)) H_{0} \leq \operatorname{dim} E([0, \varepsilon)) H=\beta,
$$

there is a nonzero vector $f \in H_{0}$ such that $E([0, \varepsilon)) f=0$. Thus, $f=$ $E([\varepsilon, \infty)) f$ and

$$
\||T| f\|=\||T| E([\varepsilon, \infty)) f\| \geq \varepsilon\|f\|
$$

which is a contradiction.

Suppose there is a subspace $H_{\varepsilon}$ of dimension $\beta$ such that $\left\|T \mid H_{\varepsilon}\right\|<\varepsilon$ and no subspace of higher dimension has this property. If $\operatorname{dim} E([0, \varepsilon)) H=\gamma<\beta$ then the argument in the preceding paragraph leads to a contradiction. It is not possible for $\gamma>\beta$ since $H_{\varepsilon}$ can be chosen to be $E([0, \varepsilon)) H$. This proves the lemma.

The next theorem shows that ess nul $T$ has a lower semicontinuous property.

5. Theorem. If $\left\|T-A_{j}\right\| \rightarrow 0$ and $\alpha=\liminf$ ess nul $A_{j}$ then ess nul $T \geq \alpha$.

Proof. Replace $A_{j}$ with a subsequence such that $\lim$ ess nul $A_{j}=\alpha$. Let $G_{j}(\cdot)$ denote the spectral measure for $\left|A_{j}\right|$. Since the values of ess nul $A_{j}$ are discrete, it follows that $\operatorname{dim} G_{j}([0, \varepsilon)) H=$ ess nul $A_{j}$ for positive $\varepsilon$ sufficiently small and that ess nul $A_{j}=\alpha$ for $j$ sufficiently large. Let $\varepsilon>0$ be given. Choose $j$ sufficiently large that $\left\|T-A_{j}\right\|<\varepsilon / 2$ and ess nul $A_{j}=\alpha$. Then choose $\gamma>0$ sufficiently small that $\gamma<\varepsilon / 2$ and $\operatorname{dim} G_{j}([0, \gamma)) H=\operatorname{ess}$ nul $A_{j}=\alpha$. Note that

$$
\left\|T\left|G_{j}([0, \gamma)) H\|\leq\| A_{j}\right| G_{j}([0, \gamma)) H\right\|+\left\|T-A_{j}\right\|<\varepsilon .
$$

By the preceding lemma we conclude that $\operatorname{dim} E([0, \varepsilon)) H \geq \operatorname{dim} G_{j}([0, \gamma)) H=\alpha$. 


\section{REMARKS}

In this section we note that a certain characterization of the closure of the invertible operators $\mathscr{G}^{-}$on a separable Hilbert space does not hold more generally. Izumino shows in [7, Corollary to Theorem 2] that $\mathscr{G}^{-}$is the set of all compact perturbations of the operators $T$ with nul $T=\operatorname{def} T$. The next result shows that Izumino's characterization does not extend to the nonseparable case. We say that $T$ has index equal to zero, written ind $T=0$, provided $\operatorname{nul} T=\operatorname{def} T$.

6. Theorem. The set of operators $T+K$ with ind $T=0$ and $K$ a compact operator is a subset of the closure of the invertible operators $\mathscr{G}^{-}$but sometimes it is a proper subset.

Proof. In order to show that $(T+K) \in \mathscr{G}^{-}$we use Izumino's argument. Let $T=U|T|$ be a polar factorization with $U$ unitary. For each positive integer $j$ we see that $\left(|T|+1 / j+U^{*} K\right)$ is a Fredholm operator with index 0 since it is a compact perturbation of an invertible operator. Thus, $U\left(|T|+1 / j+U^{*} K\right)$ is a sequence of Fredholm operators with index 0 that converges to $T+K$. It follows from Theorem 3 or Harte's theorem that any Fredholm operator with index 0 is the limit of invertible operators. Thus, $(T+K) \in \mathscr{G}^{-}$.

Now we describe an operator $B$ in $\mathscr{G}^{-}$that cannot be represented as $T+K$ with ind $T=0$ and $K$ compact. Let $R$ be multiplication by the independent variable on $H=L^{2}(d \mu)$ where $\mu$ is the counting measure on $[0,1]-$ i.e., $\mu(\mathscr{S})$ is the cardinality of $\mathscr{S}$. Note that the Schauder dimension of $H$ is $2^{\aleph_{0}}$. Let $H_{0}$ be the subspace $H \chi_{[0,5]}$ where $\chi_{[0,5]}$ is the characteristic function of the closed interval $[0, .5]$. Let $U$ be an isometry of $H$ onto $H_{0}$ and let $B=U R$. Note that nul $B=0, \operatorname{def} B=2^{\aleph_{0}}$ and, ess nul $B=2^{\aleph_{0}}=$ ess def $B$. By Theorem 3 it follows that $B \in \mathscr{G}^{-}$.

No compact perturbation of $B$ can have index zero because $\operatorname{nul}(B+K) \leq \aleph_{0}$ and $\operatorname{def}(B+K)=2^{\aleph_{0}}$ for any compact operator $K$. Both observations follow from the fact that $\operatorname{dim}(\operatorname{ker} K)^{\perp} \leq \aleph_{0}$ for any compact operator $K$. This last assertion follows from the Riesz-Schauder theory for compact operators. See [8], for example.

\section{ACKNOWLEDGMENT}

The author is grateful to Robin Harte for stimulating exchanges relevant to this work.

\section{REFERENCES}

1. F. V. Atkinson, On relatively regular operators, Acta Sci. Math. (Szeged) 15 (1953), 38-56.

2. R. Bouldin, The norm continuity properties of square roots, SIAM J. Math. Anal. 3 (1972), 206-210.

3. __ The essential minimum modulus, Indiana Univ. Math. J. 30 (1981), 513-517. 
4. J. Feldman and R. V. Kadison, The closure of the regular operators in a ring of operators, Proc. Amer. Math. Soc. 5 (1954), 909-916.

5. P. R. Halmos, A Hilbert space problem book, D. Van Nostrand, Princeton, 1967.

6. R. Harte, Regular boundary elements, Proc. Amer. Math. Soc. 99 (1987), 328-330.

7. S. Izumino, Inequalities on operators with index zero, Math. Japonica 23 (1979), 565-572.

8. K. Yosida, Functional analysis, Academic Press, New York, 1965.

Department of Mathematics, The University of Georgia, Athens, Georgia 30602 